\title{
Análisis de la cultura de ahorro de los estudiantes universitarios
}

\author{
Aldana Marybell Ramírez Rodríguez \\ aldiramirez21@ hotmail.com \\ Universidad Nacional de Pilar \\ Pilar-Paraguay \\ Cintia Vázquez Velazco \\ Ctiv06@outlook.com \\ Universidad Nacional de Pilar \\ Pilar-Paraguay
}

\section{RESUMEN}

Este trabajo describe el análisis realizado acerca de la cultura de ahorro de los estudiantes del $3^{\circ}$ al $5^{\circ}$ curso de la carrera de Contaduría Pública Nacional de la Universidad Nacional de Pilar, Paraguay, en el año 2021. Adoptó como tipo de estudio el descriptivo, con diseño observacional, transversal, prospectivo; con enfoque cuantitativo. La población estuvo constituida por 93 estudiantes, se utilizó el $76 \%$ de la totalidad para la muestra de alumnos; la técnica de recolección de datos fue la encuesta y el instrumento manejado, el cuestionario. Los hallazgos indican que los estudiantes de la educación superior encuestados poseen hábitos de ahorro, pero no en su totalidad. Existen alumnos que poseen práctica en el área de forma ocasional y otras de forma regular. Por el contrario, otros no lo realizan por falta de hábito.

Palabras clave: hábitos; ahorro; estudiantes universitarios 


\title{
Analysis of the saving culture of university students
}

\begin{abstract}
This work describes the analysis carried out on the savings culture of students from the 3rd to the 5th year of the National Public Accounting career at the National University of Pilar, Paraguay, in 2021. The descriptive study type was adopted, with an observational, cross-sectional, prospective design; with a quantitative approach. The population consisted of 93 students, $76 \%$ of the total was used for the sample of students; the data collection technique was the survey and the instrument used, the questionnaire. The findings indicate that the higher education students surveyed have savings habits, but not all of them. There are students who have practice in the area occasionally and others on a regular basis. On the contrary, others do not do it due to lack of habit.
\end{abstract}

Keywords: habits; savings; college students

Artículo recibido: 02 noviembre. 2021 Aceptado para publicación: 28 noviembre 2021 Correspondencia: aldiramirez21@ @otmail.com Conflictos de Interés: Ninguna que declarar 


\section{INTRODUCCIÓN}

El trabajo aborda el estudio de la cultura de ahorro de los estudiantes de la Carrera Contaduría Pública Nacional de la Facultad de Ciencias Contables, Administrativas y Económicas perteneciente a la Universidad Nacional de Pilar.

El interés que se generó para iniciar estudios en esta línea de investigación fue la de considerar la importancia que tiene la práctica de ahorro en la vida de las personas. Esta actividad posee diversos beneficios como: utilizarlo en el futuro para sostener una dificultad económica, reducir deudas, invertir en algún negocio, etc. (Banco central de Bolivia, 2017, p. 20).

Aponte (2017) considera que la cultura de ahorro es la base del crecimiento y es un elemento fundamental para el desarrollo, por tal razón es de suma importancia adquirir hábitos de ahorro porque permite a las personas protegerse de situaciones difíciles o inesperadas que puedan presentarse (p.52).

A su vez, la relevancia de la práctica del ahorro radica en que actúa como "uno de los principales pilares de la gestión de las finanzas personales, juega un papel preponderante en la gestión de los objetivos de los presupuestos y potencia la inversión" (López, 2016, p. 56).

\section{Problema de investigación}

En la actualidad los estudiantes universitarios pertenecen a una sociedad consumista donde la práctica de la cultura de ahorro se ve afectado por el desconocimiento que poseen los mismos acerca del buen habito de ahorro.

Los estudiantes que cursan una carrera profesional en las áreas contables-administrativas de la Facultad de Ciencias Contables, adquieren conocimientos en materia financieraadministrativa, pero no lo complementan con prácticas de uso eficiente de los recursos, estos al momento de realizar procedimientos relacionados al ingreso-egreso, no demuestran habilidades necesarias para llevar a cabo de forma eficiente los recursos económicos.

Para que los mismos implementen buenas prácticas en cuanto al manejo de dinero, ingreso, gastos, ahorros, etc., Cardozo (2019, p.2) plantea que se debe consolidar una mayor educación financiera lo cual le permitirá a los estudiantes elevar los niveles de ahorro. 
A partir de esta situación, surgió la curiosidad de analizar la cultura de ahorro en los estudiantes de la educación superior, conocer la manera de como practican los hábitos financieros. Igualmente se busca conocer si los mismos lo consideran importante en su vida diaria y para el futuro.

Por ello, la presente investigación se formó, porque se hace necesaria el estudio de la cultura de ahorro de los estudiantes universitarios, ya que el mismo permite el desarrollo, tanto para una organización como para cualquier persona que ahorra, además que ya está al alcance de todos los niveles socioeconómicos (López, 2016, p.56).

\section{El Ahorro}

Para Garzon (como se citó en Cotacallapa, 2018) el ahorro es:

Aquella cantidad separada del gasto que se destina a una meta específica. Esta meta puede ser una inversión, que es cualquier cosa que se adquiere para obtener un riesgo o beneficio futuro, con esto se está generando más riqueza (p. 31).

Por su parte, Tomala y González citado por Estrada, Arévalo, Moya, Aranda y Rivera (2017) coincide con lo expuesto por Garzon ya que define al ahorro como "el proceso mediante el cual una economía reserva parte de su producto y lo utiliza para generar ingresos en el futuro" (p.57).

Asi mismo, López (2016) afirma que "el ahorro es tomar una porción del ingreso no destinada al consumo, y conservarla en cualquier ente económico empresarial o personal para ser utilizado en el futuro" (p.33).

Comparando las definiciones presentadas por estos autores, se pudo considerar la posición común que poseen en cuánto al ahorro, todos manifiestan que el ahorro es la diferencia entre los gastos y los ingresos que se reservan para utilizarlo en el futuro ya sea para cuestiones personales o de otra índole.

\section{Capacidad de ahorro}

Actualmente hablar de la palabra ahorro "no significa gastar menos, el reto es saber cómo gastar el dinero, el ahorro es una actitud inteligente del individuo, es fácil decir que se puede hacer, pero muy difícil de hacerlo” (citado por Reus González \& Reus González, 2016, p. 86).

La capacidad de ahorro es apartar un porcentaje de los ingresos que tiene una persona con el fin de utilizarlo para beneficios futuros, pero eso no significa que al hacerlo va a 
disminuir la calidad de vida de las personas. Esto solo funciona si los egresos mensuales son inferiores a los ingresos, ya que si se gasta menos, existe la posibilidad de iniciar un ahorro (Banco Pichincha, 2021, p.2).

De acuerdo con la Comisión Nacional de Mercado de Valores y Banco (como se citó en Sumari, 2016, p. 25) "la capacidad de ahorro está directamente ligada a la reducción de las deudas. Cuando se elimine una, se debe procurar siempre destinar ese dinero al ahorro".

Basándonos en lo expuesto, en otras palabras, se puede decir que la capacidad de ahorro, realmente se desarrolla cuando se elimina una deuda y el dinero nuevo que ingresa, ya se destina al ahorro, y no para generar otra deuda, que puede ser a través de la compra de productos de alto valor o servicios (Comisión Nacional de Mercado de Valores y Banco, citado en Sumari, 2016).

Por otro lado, para Sierra (2018) las finanzas personales se relacionan con la capacidad de generar ahorro, menciona que:

Las Finanzas Personales pretenden, como punto de partida, que las personas o familias se apropien de la educación financiera, estructuren sus ingresos, ahorren e inviertan en bienes que permitan el crecimiento de la familia, tanto a nivel social como económica (p.18).

Seguidamente como antecedente investigativo aparece una parte de los resultados de la encuesta realizados por (Estrada, et al., 2017) muestra en su investigación que gran parte de los jóvenes encuestados poseen escasos conocimientos sobre el uso adecuado de sus finanzas, por lo tanto, no saben cómo ahorrar y en qué lugar hacerlo (p. 58).

Otro dato interesante que arroja esta investigación es que, los jóvenes encuestados sostienen que no pueden ahorrar por no tener ingresos suficientes al estar en dependencia de sus padres (Estrada, et al., 2017).

Teniendo en cuenta todo lo anterior, a través de este trabajo se podrá analizar la cultura de ahorro de los estudiantes universitarios, por lo tanto, lo expuesto se considera como objetivo de esta investigación.

\section{METODOLOGÍA}

Se adoptó un tipo de investigación descriptivo con enfoque cuantitativo porque busca describir características fundamentales de los sujetos de estudio referente al problema en 
cuestión (Guevara, Verdesoto \& Castro, 2020, p.2).

El diseño fue observacional, transversal y prospectivo; los datos se muestran sin intervenir en el curso natural del contexto, se aplicó en un solo momento y fueron recogidos de la fuente primaria, a medida que van sucediendo los hechos (Manterola, Quiroz, Salazar \& García, 2019).

Como técnica de recolección de datos se empleó la encuesta y el instrumento manejado fue un cuestionario desarrollado por (Estrada, et al., 2017) que cuenta con nueve ítems. Dependiendo del nivel de pregunta que se presenta, las opciones de respuestas varían.

La población de estudio estuvo constituida por 93 estudiantes de la Carrera Contaduría Pública Nacional, perteneciente al tercero, cuarto y quinto curso del año 2021. En este trabajo se utilizó el $76 \%$ de la totalidad para la muestra de alumnos que equivale a 71 estudiantes correspondientes a los cursos mencionados.

\section{RESULTADOS Y DISCUSIÓN}

Se procedió a la aplicación del cuestionario para obtener mayor conocimiento sobre la cultura de ahorro de los estudiantes, del mismo participaron 57 alumnas y 14 alumnos, a lo que respecta que se obtuvo una mayor participación de mujeres con un 80,3\% y tan solo $19,7 \%$ de participación por parte de los varones.

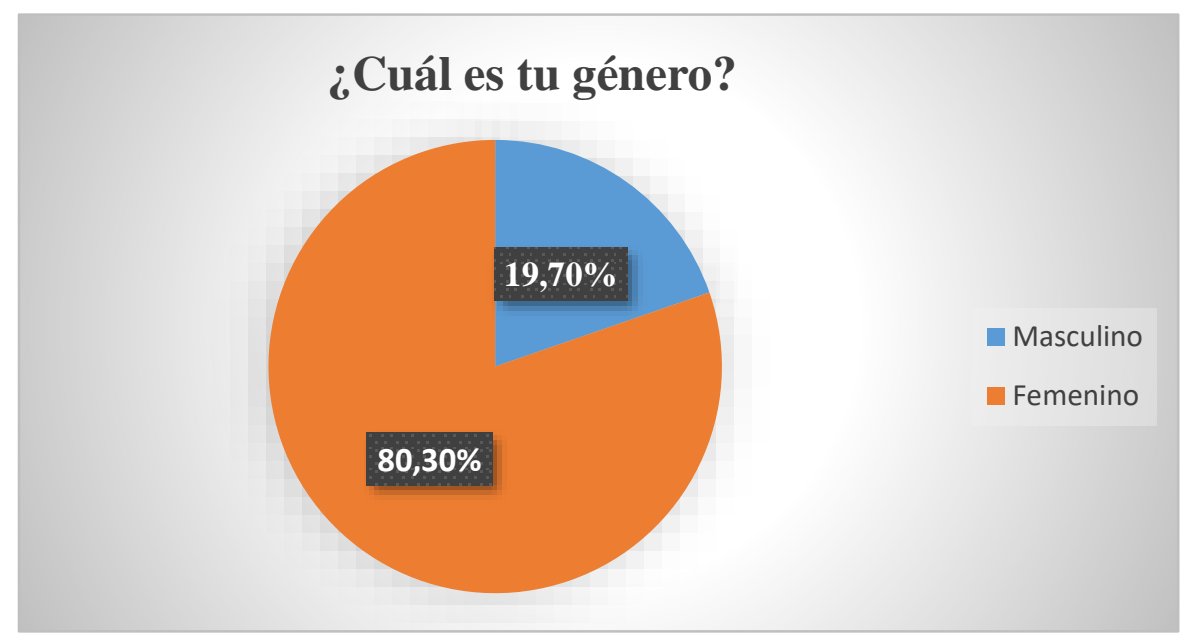

Fuente: Elaboración propio

En cuanto a los ingresos económicos dan cuenta de que el $74,6 \%$ de la población encuestada poseen ingresos económicos de alguna fuente de trabajo, sin embargo, el $25,4 \%$ no poseen ninguna fuente de ingresos. A lo que señala la Organización Internacional del Trabajo (2015) que los ingresos económicos en los hogares es de vital importancia ya que ayudan a las personas a desarrollarse social y humanamente, así 
mismo, manifiesta que la "expansión del empleo productivo es la vía hacia el crecimiento y la diversificación de las economías para que todas las personas logren un desarrollo humano y económico" (p.1).

Por otro lado, el 45\% de los estudiantes afirman que, al acceder a sus ingresos, una parte del mismo lo utilizan como ahorro, este dato es muy relevante ya que coincide con López (2016, p.33) porque el mismo manifiesta que el ahorro es tomar una parte del ingreso y conservarla para futuras necesidades, esto lleva a entender que casi la mitad de los alumnos participantes de la encuesta desarrollan la práctica del ahorro, en cambio, el $42,3 \%$ lo practican de forma ocasional, es decir que realizan el ahorro pero al termino de algunos días lo empiezan a gastar y el $12,7 \%$ de los estudiantes señalan que no realizan ahorro en su vida diaria.

Relacionando con el dato anterior, los estudiantes que no han realizado ahorro en su vida diaria confirman que no tienen dinero suficiente para ahorrar y sobrellevan muchos gastos, otra parte de los estudiantes señalan que no poseen el hábito de realizar ahorros y otro grupo menciona que dependen de sus padres, por lo tanto, consideran que no tienen la necesidad de realizar ahorros. Ante este fundamento, Cardozo (2019) revela que es necesario fortalecer una mayor educación financiera para crear hábitos y poder elevar los niveles de ahorro.

En el caso de los estudiantes que realizan ahorros, el 54, $8 \%$ consideran adecuado guardarlo en cajas de ahorro, en cambio el 41,9\% de los alumnos consideran utilizar el método tradicional el cuál es la alcancía y el 14,5\% manifiestan que lo realizan en cuentas de ahorro en bancos. Los datos obtenidos en este ítem se consideran muy importantes ya que la estrategia de ahorro depende del interés de cada persona. Según Jaramillo \& Daher (2015) “algunas se consideran predominantes, pero no exclusivos” (p. 10).

Otro ítem muestra que el 52\% de los alumnos después de realizar sus pagos, el dinero excedente lo guardan para ahorro con el fin de acrecentar el capital, por el contrario, el $29 \%$ de los alumnos revelan que al tener dinero sobrante lo gastan de forma inmediata, aparte de esto el 19\% de los mismos invierten el dinero excedente en algún negocio o proyecto. Según el Departamento de Trabajo de Estados Unidos (2019, p.6) "El desafío es no solo aprender a ahorrar sino también aprender a invertir. El conocimiento acerca de planes de ahorros y principios de inversión es clave para el crecimiento de los ahorros". De acuerdo al resultado obtenido se observa que existe un porcentaje bajo de la práctica 
de inversión entre los alumnos, en cambio, se refleja un buen porcentaje de personas que ahorran después de realizar sus pagos correspondientes.

A continuación, se presenta como algo positivo la importancia que los alumnos otorgan al conocimiento sobre finanzas personales. Casi el $60 \%$ de los alumnos consideran muy importante poseer conocimientos acerca de las finanzas, así como lo afirma Sierra (2018) "las personas o familias se deben apropiar de la educación financiera, porque eso les permitirá el crecimiento, tanto a nivel social como económica" (p.18). Por otra parte, el $37 \%$ de los mismos lo consideran importante y el 4,2\% algo importante. En términos generales todos los alumnos coinciden en que adquirir conocimientos en finanzas personales resultará valioso para el desarrollo económico personal.

Seguidamente se muestra el ítem que hace referencia a la meta principal que desean lograr los estudiantes que realizan ahorro. La mayor cantidad de porcentaje es de $46 \%$, estos consideran pagar su educación con el ahorro que van realizando, otros alumnos con un $16 \%$ manifiestan que su meta es ir de viaje. Por otro lado, el $14 \%$ revelan que desean independizarse de sus padres, así de igual manera, otros alumnos con un 14\% indican que su meta es instalar un negocio propio. Además, el 10\% expresan que ahorran para comprar algo que les gusta o les haga necesario.

Por último, se presentan los resultados de las ventajas de que una persona ahorre. Como lo manifiesta el Banco Central de Bolivia (2017, p.20) el ahorro favorece a las personas para sostener una dificultad económica, reducir deudas, invertir en algún negocio, etc. Lo dicho concuerda con lo expresado por los alumnos porque lo consideran de suma relevancia. El $73 \%$ de los estudiantes declaran que la ventaja principal es tener dinero para imprevistos, otros consideran que es importante porque no tienen que pedir prestado a otras personas o entidades, así mismo manifiestan que otra de las ventajas es contar siempre con dinero y para desarrollar algún proyecto.

\section{CONCLUSIÓN}

Este trabajo se centró en analizar la cultura de ahorro de los estudiantes de la carrera de contabilidad demostrando que la mayoría de los alumnos encuestados poseen ciertos hábitos de ahorro, es decir que algunos lo realizan para largo plazo y otros de forma ocasional y a corto plazo. Sin embargo, existen alumnos que no realizan ahorro por la razón de que no poseen hábitos financieros. 
Entre las fortalezas identificadas aparece que los alumnos priorizan como meta principal destinar su ahorro para pagar su educación, así mismo la de independizarse de sus familias y situar un negocio propio. Además, se considera positivo que exista alumnos que practican la inversión para multiplicar los ingresos sobrantes.

De la misma manera, obtener conocimientos sobre las finanzas personales resulta de suma importancia para los alumnos porque identifican su necesidad para conseguir el crecimiento económico, personal y social.

En cuanto a las debilidades, algunos de los alumnos no poseen hábitos de ahorro porque tienen muchos gastos y como consecuencia no obtienen dineros excedentes, en otros casos, en las que tengan dinero sobrante después de realizar sus pagos, lo terminan gastando de forma innecesaria por la falta de administración de sus ingresos.

Se concluye que los estudiantes de la educación superior poseen hábitos de ahorro, pero no en su totalidad. Existen alumnos que poseen práctica en el área de forma ocasional y otras de forma regular. Por el contrario, otros no lo realizan por falta de hábito.

Por lo expuesto, se insta a promover espacios de formación para que los estudiantes puedan acceder y aumentar sus conocimientos en aspectos financieros. Así como lo indica Cardozo (2019) se debe elevar los niveles de ahorro y fortalecer una mayor educación financiera para lograr los hábitos que se necesitan y obtener un buen desarrollo económico.

\section{LISTA DE REFERENCIAS}

Aponte, C. (2017). La cultura del ahorro y su importancia. Venezuela.

Banco Central de Bolivia (2017). La importancia de ahorrar en tiempos buenos para enfrentar tiempos difíciles: La experiencia con mi familia, comunidad o país. La Paz. Bolivia.

Banco Pichincha (2021). ¿Cómo conseguir la libertad financiera que tanto deseas? Clave para alcanzar la libertad financiera.

Cardozo, R. (2019). Tres consejos de educación financiera. Banco Bilbao Vizcaya Argentaria. (BBVA).

Cotacallapa, R. (2018). Análisis de la educación financiera del personal que labora en la Misión del Lago Titicaca-Puno. Universidad Peruana Unión. Escuela profesional de Contabilidad. Perú. 
Departamento de Trabajo de Estados Unidos (2019). Dinero y futuro económico: Una guía para ahorrar.

Estrada, A; Arévalo, P; Moya, S; Aranda, C y Rivera, S. (2017). Propuesta de Cuestionario para medir el nivel de conocimiento sobre las finanzas personales de jóvenes universitarios. Escuela profesional de administración de negocios globales.

Guevara, G, Verdesoto, A y Castro, N (2020). Metodologías de Investigación Educativa (descriptivas, experimentales, participativas y de investigación - acción).

Jaramillo, A y Daher, M (2015). El ahorro como estrategias de intervención social para la superación de la pobreza: estudio cualitativo sobre experiencias de ahorro de personas chilenas. Universitas Psychologica.

López, J. (2016). Las finanzas personales, un concepto que va más allá de su estructura. Universidad EAFIT. Medellín Colombia.

Manterola, Quiroz, Salazar y García (2019). Metodología de los tipos y diseños de estudio más frecuentemente utilizados en investigación clínica. Chile.

Neill, D y Cortez, L. (2018). Procesos y fundamentos de la Investigación Científica. Editorial Utmach. Machala. Ecuador.

Organización Internacional del Trabajo (2015). La importancia del empleo y los medios de vida en la agenda para el desarrollo.

Reus, N y Reus, T. (2016). El ahorro y control financiero en estudiantes del área económico - administrativo de la región altos sur de Jalisco, México. Universidad de Guadalajara.

Sierra, F. (2018). Estudio de referencia de como los estudiantes de finanzas y contabilidad de la Universidad de Jaén España están administrando los recursos a través de las finanzas personales. Universidad de Santander (UDES).

Sumari, J. (2016). Factores determinantes de la educación financiera en personas adultas de la urbanización San Santiago de la ciudad de Juliaca, periodo 2015. Universidad Peruana Unión. Juliaca-Perú. 